\title{
Cells differentiated from mouse embryonic stem cells express renal marker molecules following co-cultured with fetal renal cells
}

\author{
Li-Xing Zhang ${ }^{1}$, Yang Wang ${ }^{1}$, An Xie $^{1}$, Yuan-Lei Lou ${ }^{1}$, Yu-Xia Liu ${ }^{1}$ \\ ${ }^{1}$ Institute of Urology, First affiliated Hospital of Nanchang University, Nanchang 330006, China
}

Embryonic stem(ES) cells are known to be pluripotent becauseof their capability to differentiate into cell types of all three germ layers including germ cells. Recent studies have demonstrated that ES cells are potential to differentiate into a broad spectrum of specialized cell types, including hepatocyte, cardiomyocyte and alveolar epithelial type II cell types and even form the mature structure following co-cultured with different embryonic organic cells. Researchers have reported that ES cells could differentiated into renal lineage via embryoid bodies (EBs) in vitro, and the latest report has shown that cells isolated from that EBs formed the proximal tubules in vivo and without teratoma formation. But whether the ES cells could interact with the fetal renal cells and proceed a special differentiation hasn't been reported yet. Here, we show that ES cells differentiate into renal cell types via cocultured with mouse fetal renal cells in vitro. Mouse ES-D3 cells and fetal renal cells (E14) were co-cultured in a dual chamber dish. Renal marker molecules were detected by RT-PCR and immunofluorescent staining. Results showed that the cells differentiated from ES cells cocultured with fetal renal cells expressed marker genes characteristic for initiation of nephrogenesis (WT-1, emx-2 and Wnt-4) and terminally differentiated renal cell types (podocalyxin, Nephl and RSOR), they were coincidence with the fetal renal cells used in coculture. Cells differentiated from ES cells cultured in routine condition without mouse embryonic fibroblasts (MEF) feeder layer only expressed the Wt-1 gene. We also investigated the gene expressions of Oct-4, Nanog and Klf-4 which are associated with the ES cells' capacity to differentiate into all kinds of cells, and found that they were expressed in undifferentiated ES, but obviously depressed in differentiated cells. In summary, the fetal renal cell microenvironment may lead the ES cells differentiate toward a renal lineage, it hints the microenvironment of embryonic organic cells may play a crucial role during the transdifferentiation of ES cells.

Keywords: ES cells, nephrogenesis, differentiation, fetal renal cell

Cell Research (2008) 18:s46. doi: 10.1038/cr.2008.136; published online 4 August 2008

Correspondence: Yang Wang

E-mail: wangy63cn@sina.com 